\title{
III. Note on the essential Conditions that a Population breeding at random should be in a Stable State.
}

\author{
BY K. PEARSON, F.R.S.
}

Let us deal with bi-parental inheritance in the first place. Let $x$ be a character in the father, mean $\bar{x}$, standard deviation $\sigma_{1}$; let $y$ be the same character in the mother, $\bar{y}$ its mean, and $\sigma_{2}$ its standard deviation. Let $z$ be the character in offspring of one sex, $\sigma_{3}$ be the standard deviation of all offspring of this sex and $\bar{z}$ the mean. Let $\mu_{2}^{\prime}, \mu_{3}^{\prime}, \mu_{4}^{\prime} ; \mu_{2}^{\prime \prime}, \mu_{3}^{\prime \prime}, \mu_{4}^{\prime \prime}$; and $\mu_{2}^{\prime \prime \prime}, \mu_{3}^{\prime \prime \prime}, \mu_{4}^{\prime \prime \prime}$, be the moment coefficients about the means respectively of father, mother and offspring frequency distributions. Let $\bar{z}_{x y}$ be the mean of the offspring of those parents, who have characters $x$ and $y$, and let the array of frequency of such offspring be given by $f_{3}(u) d u$ about $\bar{z}_{x y}$, i.e. the character of any offspring in this array is $\bar{z}_{x y}+u$, where $u$ is independent of the parental characters $x$ and $y$, but $\bar{z}_{x y}$ is a function of $x$ and $y$ the parental characters. Some writers have suggested that the offspring character should be taken as a blend of the parental characters, i.e.

$$
z=\frac{1}{2}(x+y)
$$

understanding by blend the mean of the parental characters. This appears to be very unsatisfactory for:

(a) It supposes the parental characters to fix absolutely the offspring characters which is far from a result of experience.

(b) It supposes the mother to reproduce the female size of character in the male and the female offspring alike, whereas she contributes to each the sex character of her own stock, i.e. if she is a tall woman, she would contribute absolutely more to a son than to a daughter. The late Sir Francis Galton got over this difficulty by "reducing female measures to their male equivalents." This he did by altering absolute measurements in the ratio of male to female mean measurements. Thus he would take for the mean of his array of offspring

$$
\bar{z}_{x y}=\frac{1}{2}\left(x+\frac{\bar{x}}{\bar{y}} y\right)
$$

if he were dealing with male offspring. A more reasonable hypothesis is to assume that

$$
z=\frac{1}{2} \sigma_{3}\left(\frac{x}{\sigma_{1}}+\frac{y}{\sigma_{2}}\right)+u
$$

This will practically agree with Sir Francis's form, if the coefficients of variation in the two sexes are the same, i.e. $\sigma_{1} / \bar{x}=\sigma_{2} / \bar{y}$.

If we measure $u$ from the mean of the array of offspring we have

$$
\bar{z}=\frac{1}{2} \sigma_{3}\left(\frac{\bar{x}}{\sigma_{1}}+\frac{\bar{y}}{\sigma_{2}}\right)
$$

We shall now suppose the offspring to follow the law (i), or

$$
z-\bar{z}=\frac{1}{2} \sigma_{3}\left(\frac{x-\bar{x}}{\sigma_{1}}+\frac{y-\bar{y}}{\sigma_{2}}\right)+
$$

where $x$ and $y$ are uncorrelated (mating at random), and $u$ represents other influences than the parental, and is therefore uncorrelated with $x$ and $y^{*}$. The frequency distributions of $x$ and $y$

- This assumes the homoscedasticity of the arrays of offspring due to pairs of fathers and mothers with characters $x$ and $y$. 
may be taken as given by $f_{1}(x-\bar{x})$ and $f_{2}(y-\bar{y})$. Let $N_{1} \times N_{2}$ be the total number of possible matings

$$
=\iint f_{1}(x-\bar{x}) f_{2}(y-\bar{y}) d x d y
$$

and the total number of offspring $N_{3}$ in any array

$$
=\int f_{3}(u) d u \text {. }
$$

I now propose to give the expression for the $n$th moment coefficient about the mean, i.e. $\mu_{n}^{\prime \prime \prime}$, of the population of offspring of a given sex. We have

$$
N_{1} \times N_{2} \times N_{3} \times \mu_{n}^{\prime \prime \prime}=\iiint\left\{\frac{1}{2} \sigma_{3}^{n}\left(\frac{x-\bar{x}}{\sigma_{1}}+\frac{y-\bar{y}}{\sigma_{2}}\right)+u\right\}^{n} f_{1}(x-\bar{x}) f_{2}(y-\bar{y}) \times f_{3}(u) d x d y d u
$$

the integration being extended over the whole of the frequency distributions of father, mother and offspring. Thus

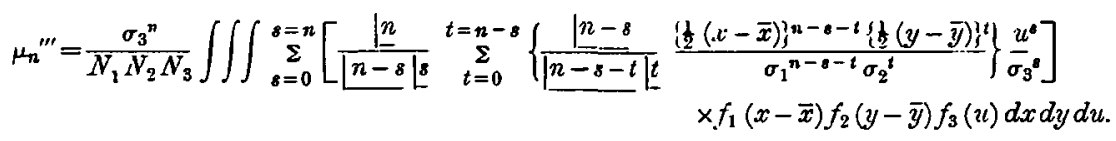

Now $x, y$ and $u$ being independent we have

$$
\begin{aligned}
& \frac{1}{N_{1}} \int(x-\bar{x})^{n-s-t} f_{1}(x-\bar{x}) d x=\mu_{n-8-t}^{\prime} \\
& \frac{1}{N_{2}} \int(y-\bar{y})^{t} f_{2}(y-\bar{y}) d y=\mu_{t}^{\prime \prime} \\
& \frac{1}{N_{3}} \int u^{8} f_{3}(u) d u=\mu_{s}^{i v} .
\end{aligned}
$$

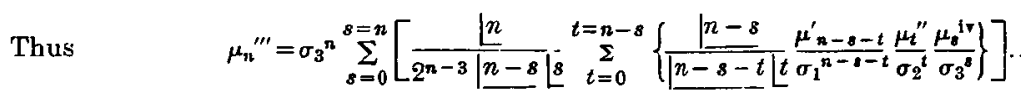

Thus we reach, remembering that $\mu_{1}{ }^{\prime}=\mu_{1}{ }^{\prime \prime}=\mu_{1}{ }^{\prime \prime}=0$,

$$
\begin{aligned}
& \mu_{2}^{\prime \prime \prime}=\frac{1}{4} \sigma_{3}^{2}\left(\frac{\mu_{2}^{\prime}}{\sigma_{1}^{2}}+\frac{\mu_{2}^{\prime \prime}}{\sigma_{2}^{2}}\right)+\mu_{2}^{i v} \\
& \mu_{3}^{\prime \prime \prime}=\frac{1}{8} \sigma_{3}^{3}\left(\frac{\mu_{3}^{\prime}}{\sigma_{1}^{3}}+\frac{\mu_{3}^{\prime \prime}}{\sigma_{2}^{3}}\right)+\mu_{3}^{\text {iv }} \\
& \mu_{4}^{\prime \prime \prime}=\frac{1}{16} \sigma_{3}^{4}\left(\frac{\mu_{4}^{\prime}}{\sigma_{1}^{4}}+6 \frac{\mu_{2}^{\prime}}{\sigma_{1}{ }^{2}} \frac{\mu_{2}^{\prime \prime}}{\sigma_{2}{ }^{2}}+\frac{\mu_{4}^{\prime \prime}}{\sigma_{2}{ }^{4}}\right)+\frac{3}{2} \sigma_{3}^{2}\left(\frac{\mu_{2}^{\prime}}{\sigma_{1}{ }^{2}}+\frac{\mu_{2}{ }^{\prime \prime}}{\sigma_{2}{ }^{2}}\right) \mu_{2}^{\mathrm{iv}}+\mu_{4}{ }^{\mathrm{Iv}}
\end{aligned}
$$

But $\mu_{2}^{\prime}=\sigma_{1}^{2}, \mu_{2}^{\prime \prime}=\sigma_{2}^{2}$, and $\mu_{2}^{\prime \prime \prime}=\sigma_{3}^{2}$. Hence we must have

$$
\mu_{2}^{i v}=\frac{1}{2} \sigma_{3}^{2}
$$

If as usual we take $\beta_{1}=\mu_{3}{ }^{2} / \mu_{2}^{3}$ and $\beta_{2}=\mu_{4} / \mu_{2}^{2}$ we find from (vi) aud (vii), writing $s^{2}=\mu_{2}{ }^{1 v}$

$$
\begin{aligned}
& \sqrt{\beta_{1}^{\text {iv }}}=\left(\frac{\sigma_{3}}{8}\right)^{3}\left\{\sqrt{\beta_{1}{ }^{\prime \prime \prime}}-\frac{1}{8}\left(\sqrt{ }^{\prime} \beta_{1}+\sqrt{\beta_{2}^{\prime}}\right)\right\} \\
& \beta_{2}{ }^{\mathrm{iv}}=\frac{\sigma_{3}^{4}}{8^{4}}\left\{\beta_{2}{ }^{\prime \prime \prime}-\frac{1}{16}\left(\beta_{2}{ }^{\prime}+\beta_{2}{ }^{\prime \prime}+6\right)-3 \frac{\sigma_{2}{ }^{2}}{8^{2}}\right\}
\end{aligned}
$$

Whence by the use of (viii)

$$
\begin{aligned}
& \sqrt{{\overline{\beta_{1}}}^{\text {iv }}}=2 \sqrt{ } 2\left\{\sqrt{{\overline{\beta_{1}}}^{\prime \prime \prime}}-\frac{1}{8}\left(\sqrt{\beta_{1}^{\prime}}+\sqrt{\overline{\beta_{1}{ }^{\prime \prime}}}\right\}\right. \\
& \beta_{2}^{\text {iv }}=4\left\{\beta_{2}{ }^{\prime \prime \prime}-\frac{1}{16}\left(\beta_{2}^{\prime}+\beta_{2}^{\prime \prime}+6\right)\right\}-6 \ldots
\end{aligned}
$$




\section{Miscellanea}

Hence in order that the offspring population should be stable, it is needful that in the array of offspring for given parents :

(a)

$$
s=\frac{1}{\sqrt{2}} \sigma_{3} \text {. }
$$

(b) $\quad \sqrt{\beta_{1}^{11}}=2 \sqrt{2}\left\{\sqrt{{\beta_{1}^{\prime \prime \prime}}^{\prime \prime}}-\frac{1}{8}\left(\sqrt{\hat{\beta}_{1}^{\prime \prime}}+\sqrt{\beta_{1}^{\prime \prime}}\right)\right\}=2 \sqrt{2} \sqrt{\beta_{1}^{\prime \prime \prime}}\left(1-\frac{1}{4}\right)=\frac{3 \sqrt{2}}{2} \sqrt{\beta_{1}^{\prime \prime \prime}}$,

if $\beta_{1}^{\prime \prime \prime}=\beta_{1}^{\prime}=\beta_{1}^{\prime \prime}$, i.e. the skewness be the same for fathers, mothers and offspring.

$$
\beta_{2}^{i v}=\frac{1}{2}\left(7 \beta_{2}^{\prime \prime \prime}-15\right)
$$

if

$$
\beta_{2}^{\prime \prime \prime}=\beta_{2}^{\prime}=\beta_{2}^{\prime \prime} \text {. }
$$

Thus, we have for the array of offspring of given parents

$$
\left.\begin{array}{rl}
s & =\frac{1}{\sqrt{2}} \sigma_{3} \\
\beta_{1}^{i v} & =\frac{9}{2} \beta_{1}^{\prime \prime \prime} \\
\beta_{2}^{1 v-}-3 & =\frac{7}{2}\left(\beta_{1}^{\prime \prime \prime}-3\right)
\end{array}\right\}
$$

Accondingly the variability of the array is less than that of the population of offspring; and the array (unless $\beta_{1}^{\prime \prime \prime}=0, \beta_{1}^{\prime \prime \prime}=3$ ) is more skew and has greater kurtosis than the general population.

If $r_{12}, r_{23}, r_{31}$ be the three correlations of father, nother and offspring we know that the wean standard-deviation of the offspring of arrays having the same parents is

and this equals if there be wo assortative mating

$$
s^{\prime}=\sigma_{3} \sqrt{\frac{1-r_{13}{ }^{2}-r_{23}{ }^{2}-r_{12}{ }^{2}+2 r_{12} r_{19} r_{31}}{1-r_{12}{ }^{2}}},
$$

$$
\left(r_{12}=0\right), \quad \sigma_{3}, \sqrt{1-r_{13}^{2}-r_{23}^{2}} \text {. }
$$

If we could assume this cqual to 8 we must have, since

leading to

$$
\begin{gathered}
8=\frac{l}{\sqrt{2}} \sigma_{3}, \\
\frac{1}{\sqrt{2}}=\sqrt{\frac{1-r_{13}^{2}-r_{23}{ }^{2}}{r_{13}+r^{2}=1}}
\end{gathered}
$$

or if the two parental currelations are equal to

$$
r_{13}=r_{23}=\cdot 5 \text {. }
$$

In other words, if the parental influences were equal and there were no assortative mating and the charncter in the array of offspring had the mean value

then the population could only be stable if

$$
\frac{1}{2} \sigma_{3}\left(\frac{x}{\sigma_{1}}+\frac{y}{\sigma_{2}}\right)
$$

$$
r_{13}=r_{23}=0.5 \text {. }
$$

But this apparently noteworthy result only hegs the question. By the general theory of correlation the mean of the array of offspring is

if there be no assortative mating,

$$
\begin{aligned}
& \bar{z}+\sigma_{3}\left(\frac{r_{13}-r_{12} r_{23}}{1-r_{12}{ }^{2}} \frac{x-\bar{x}}{\sigma_{1}}+\frac{r_{23}-r_{12} r_{13}}{1-r_{12}{ }^{2}} \frac{y-\bar{y}}{\sigma_{2}}\right), \\
= & \bar{z}+\sigma_{3}\left(r_{13} \frac{x-\bar{x}}{\sigma_{1}}+r_{23} \frac{y-\bar{y}}{\sigma_{2}}\right)
\end{aligned}
$$

$$
=\sigma_{3}\left(r_{13} \frac{x}{\sigma_{1}}+r_{23} \frac{y}{\sigma_{2}}\right)+\bar{z}-\sigma_{3}\left(\frac{r_{13} \bar{x}}{\sigma_{1}}+\frac{r_{23} \bar{y}}{\sigma_{2}}\right) .
$$

Biometrika x 
Hence if we assume the mean of array of offspring to be given by

$$
\sigma_{3} \frac{1}{2}\left(\frac{x}{\sigma_{1}}+\frac{y}{\sigma_{2}}\right)
$$

(i) the second portion of the expression must be zero, i.e. mean of whole population of offspring must coincide with mean of array of oftspring where parents have the mean values and (ii) we must have $r_{13}=r_{23}=\frac{1}{2}$. In other words the form of our assumption involves both the equal infuence of the parents and the value of the parental correlation.

From the standpoint of heredity no such assumption is legitimate. Neither in Mendelian theory nor in biometric formula, nor again in actual observation is it permissible to suppose that the mean of the array of offspring is determined solely by the parents. Still less is it possible to suppose the actual character of the offspring to be the mean of that of the parents (i.e. put $u=0$ ). If it were we should have $z=\frac{1}{2}(x+y)$, whence flow

$$
\left.\begin{array}{l}
\mu_{2}^{\prime \prime \prime}=\frac{1}{4}\left(\mu_{2}{ }^{\prime}+\mu_{2}{ }^{\prime \prime}\right) \\
\mu_{3}^{\prime \prime \prime}=\frac{1}{8}\left(\mu_{3}{ }^{\prime}+\mu_{3}{ }^{\prime \prime}\right) \\
\mu_{4}{ }^{\mathrm{iv}}=\frac{1}{16}\left(\mu_{4}{ }^{\prime}+6 \mu_{2}{ }^{\prime} \mu_{2}^{\prime \prime}+\mu_{4}^{\prime \prime}\right)
\end{array}\right\}
$$

But these equations assume that $\mu_{2}^{1 v}, \mu_{3}^{1 v}$ and $\mu_{1}^{17}$ are all zero-an absurdity in itself and contrary to all experience, whether biometric or Mendelian. For non-assortative mating and equal potency of parents, they lead to parental correlations of the order 7 and to an impossibility of stability in any population*.

In fact any such relations as (xiv) are inconceivable on the basis of both biometric as well as Mendelian theory and observation. Parental correlations have never been obserred anywhere near such a value as 0.7. Equations (xiii) are, however, suggestive; they show that if the parental distribution be symmetrical and mesokurtic, the array of offspring will remain so after selection; but if the parental distribution does not possess these characters, then any selection of individual parents will emphasize the asymmetry and the kurtosis in the resulting array of offspring ; or continued selection of this type will lead to greater and greater divergence from the normal or Gaussian frequency distribution.

* If we assume that the mean of the array of offspring of parents of characters $x$ and $y$ is given by $l x+m y$, it is only another way of asserting that the regression is linear and that

$$
l=\frac{r_{12}-r_{13} r_{23}}{1-r_{23}} \frac{\sigma_{3}}{\sigma_{1}}, \quad m=\frac{r_{13}-r_{12} r_{23}}{1-r_{33}^{2}} \frac{\sigma_{3}}{\sigma_{2}}
$$

If we make $l=m$, or give equal weight to the parents, it is only rational to suppose that $\sigma_{1}=\sigma_{2}$ and $r_{12}=r_{13}$, which lead us to

Hence the mean of the array is

$$
\begin{gathered}
l=m=\frac{r_{12}}{1+r_{23}} \frac{\sigma_{3}}{\sigma_{1}} . \\
\frac{r_{12}}{1+r_{23}} \frac{\sigma_{3}}{\sigma_{1}}(x+y),
\end{gathered}
$$

and whether we make $x$ constant and $y$ constant or $x+y$ constant leads to precisely the same rariability in the array, i.e.

$$
s=\sigma_{3} \sqrt{\frac{1-r_{12^{2}-r_{13}^{2}-r_{23}{ }^{2}+2 r_{12} r_{13} r_{23}}^{1-r_{23}^{2}}}{12}}=\sigma_{3} \sqrt{1-\frac{2 r_{12} 2^{2}}{1+r_{23}}} .
$$

If assortative mating be zero, this equals

$$
\sigma_{3} \sqrt{1-2 r_{12}^{2}}
$$

and, if to reach the results for $\mu_{2}^{\prime \prime \prime}$ given above we put this zero, we must have

$$
r_{12}=\sqrt{\cdot 50}=0.7 \text { nearly. }
$$

